\title{
Comet Shoemaker-Levy 9 Dust Size and Velocity Distributions
}

\author{
Joseph M. Hahn \\ Lunar and Planetary Institute, 3600 Bay Area Boulevard, Houston, TX 77058 \\ email: hahn@lpi.usra.edu \\ phone: 281-486-2113 \\ fax: 281-486-2162 \\ Terrence W. Rettig \\ Department of Physics, University of Notre Dame, Notre Dame, IN 46556 \\ email: trettig@nd.edu \\ phone: 219-631-7732 \\ fax: 219-631-5952
}

To appear in Icarus 


\begin{abstract}
Pre-impact observations of Comet Shoemaker-Levy 9 obtained with the Hubble Space Telescope are examined, and a model of an active, dust-producing comet is fitted to images of fragments G, H, K, and L. The model assumes steady isotropic dust emission from each fragment's sunlit hemisphere. Best-fit results indicate that the dominant light-scatterers in these fragments' comae were relatively large dust grains of radii $10 \mu \mathrm{m} \lesssim R \lesssim 3 \mathrm{~mm}$. The fragments' dust size distributions were rather flat in comparison to other comets, $d N(R) \propto R^{-2.3 \pm 0.1}$, and the dust ejection speeds were $\sim 0.5-1.5 \mathrm{~m} / \mathrm{sec}$. The S-L 9 fragments themselves were not detected directly, and upper limits on their radii are 1.0-1.5 km assuming an albedo $a=0.04$. However these fragments' vigorous production of dust, which ranges from 6-22 kg/sec, places a lower limit of $\sim 100$ meters on their radii at the moment of tidal breakup. Any fragments smaller than this limit yet experiencing similar mass loss rates would have dissipated prior to impact. Such bodies would fail to leave an impact scar at Jupiter's atmosphere, as was realized by fragments $\mathrm{F}, \mathrm{J}, \mathrm{P}_{1}, \mathrm{P}_{2}, \mathrm{~T}$, and U.
\end{abstract}




\section{Introduction}

Owing to an extremely close encounter with Jupiter on July 7, 1992, Comet Shoemaker-Levy 9 (S-L 9) tidally disrupted into approximately 21 cometary fragments. Tidal disruption effectively turned S-L 9 "inside out", which permitted a rare examination of the interior of a comet. Of course, comet observations generally provide information only about its smallest constituents, namely, the dust grains and gas molecules that manage to escape from a nucleus that usually remains hidden from view due to the surrounding coma. Although this study is no exception to this difficulty, we shall nonetheless manage to draw some conclusions about the tidally disrupted S-L 9 fragments via a close examination of their dusty comae. In particular, a model of a cometary dust coma and tail is fitted to Hubble Space Telescope (HST) observations of S-L 9 in order to extract the comet's dust size and velocity distributions, as well as to place constraints on the size of the fragments themselves.

Although Comet S-L 9 was monitored regularly since its discovery in March 1993, we shall examine only the highest resolution observations that were acquired between January 1994 (when the comet was not far from apojove) and July 1994, just prior to impact with Jupiter (see (Weaver et al. 1995)). This analysis shall concentrate on the five bright fragments G, H, K, L, and $\mathrm{S}$ that were observed on several occasions when both far from and near to Jupiter. Relevant parameters for these observations are given in Table I. Comet S-L 9 was imaged with the HST Wide Field Planetary Camera 2 using the F702W filter, which is roughly and R filter having a bandpass of 6000 to $8100 \AA$. Comet S-L 9 was observed at a geocentric distance of about 5 AU, so each $0.1^{\prime \prime}$ pixel in the Wide Field Camera subtends about $400 \mathrm{~km}$. Exposures typically lasted about 30 minutes. The data were reduced in the standard manner, with the bias and dark currents subtracted, the images flatfielded and background subtracted, and cosmic ray hits repaired. For further details see (Hahn et al. 1996).

Spectroscopic observations of S-L 9 did not detect any signatures of cometary gas emissions ((Cochran et al. 1994), (Weaver et al. 1995)). Nonetheless, the inferred upper limit for water production is consistent with non-rotating comet fragments that are smaller than about $15 \mathrm{~km}$ across ((Weissman 1996)). Although direct evidence for any gas production is absent, the emission of dust entrained by an unseen gas outflow is the most promising explanation for the Comet's appearance ${ }^{1}$. Thus a simple model of an active, dust producing comet is investigated in Section 2. Synthetic dust-coma images are generated in Section 3 and fitted to the HST observations of S-L 9. Section 4 reports results for the S-L 9 dust size and velocity distributions and also places constraints on

\footnotetext{
${ }^{1}$ Note that the early S-L 9 literature assumed that the Comet was largely inactive, and that each fragment's dust halo was instead composed of large dust created during the tidal breakup event ((Weaver et al. 1994), (Sekanina et al. 1994)). However this scenario cannot account for the observed coma-morphology since an initial dust halo would have subsequently evolved into an elongated structure having isophotes that would steadily lengthen and narrow along over time, concurrent with the growing separations among the fragments themselves ((Weissman 1996), (Rettig and Hahn 1997), (Tanigawa et al. 1997)).
} 
the fragment sizes. Section 5 then synthesizes our findings for this tidally disrupted comet.

\section{The Dust Coma/Tail Model}

The task at hand is to determine the rates at which a given S-L 9 fragment produced dust grains of various radii $R$ as well as their ejection velocities $V(R)$. We begin by defining $N(R, t)$ as the cumulative number of all dust grains having radii smaller than $R$ emitted up until some time $t$. The differential dust production rate of all grains within the size interval $R \pm d R / 2$ is written as

$$
\frac{d}{d t} \frac{d N(R, t)}{d R} \times d R
$$

or more simply $d \dot{N}(R, t)$, which is one of the quantities to be extracted from the HST observations. However it is usually quite difficult to uniquely disentangle the dust size distribution $d \dot{N}(R, t)$ from the dust emission velocity distribution $V(R, t)$ without first making some simplifying assumptions.

The standard approach is to assume that the dust size and velocity distributions obey simple power-laws which then permits their characterization in terms of a few parameters that might vary with time. However S-L 9 maintained a nearly constant distance from the Sun (Table I), and when far from Jupiter all the fragments considered here exhibited surface brightness profiles consistent with steady dust production ((Hahn et al. 1996), (Rettig and Hahn 1997)). This suggests that these fragments' dust production rates did not vary dramatically during the observation span considered here. For these reasons the remainder of this analysis assumes the dust size distribution $d \dot{N}(R)$ and outflow velocity distribution $V(R)$ were constant over time. This makes any a priori power-law assumptions unnecessary. But in order to make the problem computationally tractable, the model comet shall only emit dust having discrete radii $R_{i}=0.316 \mu \mathrm{m}, 1.0 \mu \mathrm{m}, \ldots, 3.16 \mathrm{~mm}, 1$ $\mathrm{cm}$ (i.e., with sizes incrementing every half-decade) rather than a continuous range of dust sizes. This analysis thus seeks the approximate S-L 9 dust distribution $d \dot{N}\left(R_{i}\right)$ and velocity distribution $V\left(R_{i}\right)$ evaluated at ten discrete size bins. The permitted dust ejection velocities are similarly discrete over $0.25 \mathrm{~m} / \mathrm{sec}$ intervals, with velocities ranging from 0 up to $3.5 \mathrm{~m} / \mathrm{sec}$ in fifteen velocity bins. The dust size and velocity bins considered here are chosen to span the range of sizes and velocities reported previously ((Hahn et al. 1996), (Rettig et al. 1996)), and the number of bins used here reflects a reasonable balance between the model's precision and the cost in CPU time, which increases geometrically with the number of bins.

The model comet nucleus and its dust grains are subject to solar and jovian gravities, with the dust also perturbed by solar radiation pressure. The bulk of the S-L 9 dust were evidently larger that the wavelength of visible light ((Hahn et al. 1996)), so the ratio of radiation pressure to the solar acceleration is simply $\beta=3 L_{\odot} Q_{r} / 16 \pi G M_{\odot} c \rho_{d} R$ in the geometric optics limit ((Burns et al. 1979)), where $L_{\odot}$ is the solar luminosity, $Q_{r}$ is the radiation pressure efficiency, $c$ the speed of light, $\rho_{d}$ the grain density, and $R$ the grain radius. Cometary dust as well as the surfaces of nuclei are very dark and efficient absorbers of light ((Hanner and Newburn 1989), 
(Weissman et al. 1989)), so setting $Q_{r}=1$ is a good approximation. For simplicity this analysis shall assume that the unknown density of the S-L 9 dust is $\rho_{d}=1 \mathrm{gm} / \mathrm{cm}^{3}$; if an alternate density is preferred then the grain sizes quoted herein should be rescaled by by a factor of $\rho_{d}^{-1}$. Thus $\beta \simeq 0.585(R / 1 \mu \mathrm{m})^{-1}$ provided $R$ exceeds about $1 \mu \mathrm{m}$. For grains smaller than $R=1 \mu \mathrm{m}$ we adopt a value for $\beta$ computed via Mie light-scattering theory for an "ideal" grain that absorbs only the incident sunlight that is of a wavelength shorter than the grain diameter (see Fig. (7b) of (Burns et al. 1979) and adjust for the $\rho_{d}=1 \mathrm{gm} / \mathrm{cm}^{3}$ grain density employed here).

The equations of motion for the heliocentric trajectory $\mathbf{r}(t)$ of a cometary nucleus/dust grain subject to solar and jovian gravities as well as solar radiation pressure is

$$
\ddot{\mathbf{r}}=-\frac{G M_{\odot}(1-\beta) \mathbf{r}}{r^{3}}-\frac{G M_{J}\left(\mathbf{r}-\mathbf{r}_{J}\right)}{\left|\mathbf{r}-\mathbf{r}_{J}\right|^{3}},
$$

where $M_{J}$ is the mass of Jupiter, and $\beta$ set to zero when calculating the motion of the nucleus. Jupiter's trajectory $\mathbf{r}_{J}(t)$ is computed using the DE403 JPL Planetary and Lunar Ephemeris software ((Standish et al. 1995)). Gravities due to the other planets as well as the the Sun's barycentric motion are ignored. Since Eq. (2) does not include comet gravity, the dust velocities reported here should be understood as their speed upon leaving the fragment's gravitational sphere of influence. Also neglected are any possible Lorentz forces that may have acted upon the grains as they neared Jupiter's magnetosphere during the 'last-look' imaging of July 1994.

It is necessary to simulate dust production prior to the first observations of S-L 9, so we start with the position and velocity of fragment H on July 13.01993 (near apojove) provided by Paul Chodas (1995, private communication) and integrate the fragment's motion backwards in time until just after the moment of tidal breakup using a Bulirsh-Stoer integrator ((Press et al. 1992)). The comet fragment is then integrated forwards in time until impacting Jupiter two years later. During the comet's final orbit about Jupiter, standard Monte Carlo techniques ((Lux and Koblinger 1991)) are used to emit dust grains from the comet at uniformly random times in uniformly random directions across its sunward-facing hemisphere. If a dust grain is emitted at time $t_{e}$ at velocity $\mathbf{V}$, the comet's heliocentric position $\mathbf{r}\left(t_{e}\right)$ and the grain's velocity $\dot{\mathbf{r}}\left(t_{e}\right)+\mathbf{V}$ are employed as initial conditions for Eq. (2), which is then used to advance the grain's coordinates in time to each of the observation dates listed in Table I.

We then calculate the fragment and dust grain's relative positions as seen from the vantage of Earth on each of the observation dates. The fragment and dust grain's position vectors are first translated to a geocentric coordinate system, $\mathbf{r} \rightarrow \mathbf{r}-\mathbf{r}_{\oplus}$, where $\mathbf{r}_{\oplus}$ is the vector pointing from the Sun to Earth obtained with the JPL Ephemeris software. The coordinate system is then rotated such that one Cartesian axis points at the fragment, with the remaining two sky-plane coordinate axes aligned with the rows and columns of the HST CCD images of the comet. For each run of the simulation, the model comet emits numerous dust grains having a single radius $R_{i}$ emitted at a single velocity $V\left(R_{i}\right)$ by fragment $\mathrm{H}$ during its final orbit about Jupiter. A dust column density map is then constructed for each observation date by counting all the grains subtended by each pixel in a synthetic CCD array. The $0.1^{\prime \prime}$ spatial scale of each pixel matches the sampling of the 
detector in the Wide-Field camera, and the resulting synthetic images are centered on the S-L 9 fragment and measure $20^{\prime \prime}$ across. (There is little need to model the dust tails beyond about $10^{\prime \prime}$ of the comae photocenters since the tails' signal/noise ratios have dropped to $\sim 1$ beyond this distance.) The production of $\sim 10^{6}$ grains over the comet's two-year orbit was more than sufficient to generate Monte Carlo dust tail models having statistical fluctuations much smaller than was observed in the S-L 9 data. In Section 3, the contributions by grains of various sizes $R_{i}$ and speeds $V\left(R_{i}\right)$ will be coadded in order to assemble a more realistic image of a cometary dust coma and tail.

Computation of the five images requires about ten hours of CPU time on an IBM RS6000, and this is repeated 150 times - once for each of the ten dust size bins for grains emitted at the fifteen different velocities. Finally, the images are convolved with the HST point spread function (PSF) to account for light diffraction caused by the telescope's optical system. The model PSF used here is computed with Tiny Tim, a standard software package used to generate PSFs for HST data ((Krist 1994)). Example model images are provided in Fig. 1 which shows contour maps for an $R_{i}=300 \mu \mathrm{m}, V\left(R_{i}\right)=0.75 \mathrm{~m} / \mathrm{sec}$ model on two dates. Although these images were generated specifically for the observations of fragment $\mathrm{H}$, they will also be used to fit images of the other fragments since they all followed the same path into Jupiter; except when imaged just prior to impact, the observed fragments were nearly equidistant from Jupiter.

\section{Dust Coma Fitting}

The resulting dust column density images generated in Section 2 are divided by the model's dust production rate so that each image now represents the column density of a comet having 'unit' dust production rate. These column density maps shall be denoted as $\varrho\left(x, R_{i}, V\left(R_{i}\right), t\right)$ where $x$ represents a particular pixel in the CCD image of dust having radii $R_{i}$ emitted at velocity $V\left(R_{i}\right)$ and observed at time $t$. The dust column density contributed by a single grain size bin is $\varrho\left(x, R_{i}, V\left(R_{i}\right), t\right) \times d \dot{N}\left(R_{i}\right)$, so the total flux density of sunlight reflected by the model comet is simply

$$
\begin{aligned}
F(x, t) & =\int_{R} \varrho(x, R, V(R), t) f(R, t) d \dot{N}(R)+\operatorname{PSF}(x) f\left(R_{f}, t\right) \\
& \simeq \sum_{R_{i}} \varrho\left(x, R_{i}, V\left(R_{i}\right), t\right) f\left(R_{i}, t\right) d \dot{N}\left(R_{i}\right)+\operatorname{PSF}(x) f\left(R_{f}, t\right),
\end{aligned}
$$

where $f(R, t)$ is the flux density of sunlight reflected by a grain of radius $\mathrm{R}$, and the sum is over all the grain size bins. The comet nucleus itself will also reflect sunlight, so the term on the right represents the light contributed by an S-L 9 fragment of radius $R_{f}$. Here, $\operatorname{PSF}(x)$ is the normalized PSF centered at the origin which accounts for how the light from an unresolved point source (the fragment) is distributed at the image plane.

The flux density of sunlight (here, power per area per wavelength interval) reflected by a 
spherical grain of radius $\mathrm{R}$ toward an observer a distance $\Delta(t)$ away is ((Lester et al. 1979))

$$
f(R, t)=\left(\frac{R}{\Delta(t)}\right)^{2} a \psi(\phi) f_{i n c}
$$

where $a$ is the grain's geometric albedo, $\psi(\phi)$ is its phase law, and $f_{\text {inc }}$ is the incident monochromatic solar flux density. The S-L 9 dust albedo cannot be determined from these observations, so the value $a=0.04$ measured for comet Halley ((Lorenzetti et al. 1987)) shall be adopted here. It should be noted that comet dust albedos generally range from 0.02 to 0.1 ((Hanner and Newburn 1989)). Cometary dust generally obey the empirical phase law ((Meech and Jewitt 1987))

$$
\psi(\phi)=10^{-\beta|\phi(t)| / 2.5}
$$

where $\phi(t)$ is the Sun-Comet-Earth phase angle given in Table I and the phase coefficient $\beta$ describes how the grain's reflectivity drops with phase angle. The incident solar flux density in Eq. (4) is $f_{\text {inc }}=\pi\left(R_{\odot} / r\right)^{2} B_{\lambda}$ where $R_{\odot}$ is the radius of the Sun and $B_{\lambda} \simeq 2.12 \times 10^{6} \mathrm{ergs} / \mathrm{cm}^{2} / \mathrm{sec} / \AA$ is the monochromatic solar radiance for a $T=5780 \mathrm{~K}$ blackbody at the effective wavelength $\lambda=6913$ $\AA$ of these broadband HST observations. The comet fragment also reflects sunlight as per Eq. (4) but it may have an albedo $a_{f}$ and phase law $\psi_{f}$ distinct from the dust. The albedo of cometary nuclei generally ranges from 0.02 to 0.2 ((Weissman et al. 1989), (Tokunaga et al. 1992)). The Halley nucleus albedo, which is also $a_{f}=0.04$ ((Sagdeev et al. 1986)), shall be assumed for the S-L 9 fragments. While the appropriate phase law for comet nuclei is unknown, it seems reasonable to treat the fragments as diffuse reflectors of light for which Lambert's law of reflection applies. In this case $\psi_{f} \simeq 1$ for the small phase angles considered here.

Thus $n=22$ parameters specify a single dust comet model: a $d \dot{N}\left(R_{i}\right), V\left(R_{i}\right)$ pair for each of the ten dust size bins, the phase coefficient $\beta$, and the fragment radius $R_{f}$. The dust model is adjusted to the five observations of each fragment by iteratively choosing trial solutions using the downhill simplex algorithm ((Nelder and Mead 1965), (Parkinson and Hutchinson 1972), (Press et al. 1992)) which minimizes the fit's $\chi^{2}$. Data pixels polluted by background stars, adjacent cometary comae, or an inordinate amount of spatially varying background flux are flagged and are not considered in these fits. As with all numerical minimization routines, this method only provides a solution that locally minimizes the $\chi^{2}$ along the search path through parameter space, so fits starting with numerous different trial solutions must be performed. Experience has shown that repeating the parameter search using $\sim 1000$ widely distinct trial solutions is more than sufficient

to determine the set of parameters that minimizes the fit's $\chi^{2}$. Best fits reported in Figs. 2-10.

\section{Results}

The time-sequence of contour maps of fragment $G$ are shown in Fig. 2 as well as the synthetic images fitted to the observations. Although slight discrepancies exist between the observed and the model isophotes at the fainter light levels, there is overall good agreement between the observations 
and the fit. Figure 3 gives the differential dust production rate $d \dot{N}(R)$ as well as its mass loss distribution $d \dot{M}(R)=\frac{4 \pi}{3} \rho_{d} R^{3} d \dot{N}(R)$ obtained for fragment $\mathrm{G}$, with the arrows indicating that only upper limits on the dust production rates are obtained for the corresponding size bins. These findings are typical of many comets in that production of the smallest grains are numerically favored yet the total mass loss rate is governed by the largest grains ejected. The mass loss rate of grains detected in fragment G's comae $(10 \mu \mathrm{m} \lesssim R \lesssim 3 \mathrm{~mm})$ is $\dot{M}=22 \pm 5 \mathrm{~kg} / \mathrm{sec}$.

Radiation pressure segregates grains according to their size $\times$ density, and this is illustrated by the network of syndynes and synchrones shown in Fig. 2. A syndyne is the trajectory of dust having a specified radius $R$ emitted at zero velocity at various times $t$, while synchrones trace zero-velocity dust of various radii $R$ that are all emitted at a given time $t$. Dust of radius $R$ emitted at velocity $V>0$ thus inhabit a three-dimensional envelope that surrounds the appropriate syndyne. The $R<3 \mu \mathrm{m}$ syndynes shown in the May 18 image, which was acquired very near solar opposition, confirms our earlier findings in that this fragment did not produce detectable quantities of dust much smaller than about $\sim 5 \mu \mathrm{m}$ ((Hahn et al. 1996)). Had $R=1 \mu \mathrm{m}$ dust been abundant, they would have produced a bright, narrow tail in the May 18 image extending about 7 arcseconds east of the coma photocenter. The dearth of any detectable dust beyond $\sim 5$ arcseconds to the east instead puts an upper limit of $\sim 70 \mathrm{gm} / \mathrm{sec}$ on the production rate of grains $3 \mu \mathrm{m}$ and smaller by fragment G (see Fig. 3). This is well below the limit previously established by Sekanina (1996a) based on the S-L 9 tail orientations. Note also that only an upper limit on the production of large cm-sized grains has been determined. Whether an S-L 9 fragment could actually have launched such large particles is doubtful. At S-L 9's heliocentric distance, gas-flow models show that water production is too feeble to loft grains larger than about 1 micron from the surface of a $\mathrm{km}$-sized comet fragment ((Weissman 1996)).

However a more volatile species such as CO could very well be responsible for lofting the large dust grains seen at S-L 9. Consider comets Schwassmann-Wachmann 1 and Hale-Bopp, both of which produced CO at rates of $Q \sim 3 \times 10^{28}$ molecules/sec while at $r \sim 6$ AU ((Senay and Jewitt 1994), (Biver et al. 1996)). Both comet nuclei have similar radii, $R_{n} \sim 20 \mathrm{~km}$ ((Meech et al. 1993), (Weaver and Lamy 1997)), so their surface production is $Z=Q / 2 \pi R_{n}^{2} \sim 10^{15}$ molecules $/ \mathrm{cm}^{2} / \mathrm{sec}$ assuming sunward emission. It is reasonable to expect even more vigorous $\mathrm{CO}$ production by the S-L 9 fragments due to the very recent exposure of their icy surfaces and their closer proximity to the Sun. The largest grain that can be lofted by a CO outflow is obtained by equating a fragment's surface gravity to the gas drag force, which yields $R_{\max }=9 C_{D} m_{\mathrm{CO}} Z v / 32 \pi G R_{f} \rho_{p} \rho_{d}$ where $C_{D}=2$ is the drag coefficient for free molecular flow, $m_{\mathrm{CO}}$ is the mass of a CO molecule, and $G$ is the gravitation constant ((Gombosi et al. 1986), (Hahn et al. 1996)). The gas velocity is $v=\sqrt{3 k T / m_{\mathrm{CO}}} \sim 0.4 \mathrm{~km} / \mathrm{sec}$ where $k$ is the Boltzmann constant and a blackbody temperature of $T=170 \mathrm{~K}$ is adopted at the fragment's surface. Assuming the fragment's have radii $R_{f} \sim 0.3 \mathrm{~km}$ (see Section 4.2) and internal densities of $\rho_{f} \sim 0.6 \mathrm{gm} / \mathrm{cm}^{3}$ with dust densities of $\rho_{d} \sim 1 \mathrm{gm} / \mathrm{cm}^{3}$, the largest coma grain has a radius $R_{\max } \sim 3 \mathrm{~mm}$. Of course the good agreement between $R_{\max }$ and the largest dust-grain detected at fragment G (see Fig. 3) is merely accidental since most of 
the quanties adopted above are quite uncertain.

The grain size distribution for fragment $\mathrm{G}$ is also rather unique. The logarithmic slope of $d \dot{N}(R)$ in Fig. 3 over the $10 \mu \mathrm{m} \lesssim R \lesssim 3 \mathrm{~mm}$ size interval indicates $d \dot{N}(R) \propto R^{-a}$ with $a=2.3 \pm 0.1$. The color of comet dust is also sensitive to the slope of the dust size distribution, and recent fits to the S-L 9 photometry favor a dust size power-law that is even shallower than $a \sim 2$ ((Tanigawa et al. 1997)). Note however that these size distributions are considerably flatter than the $R^{-3.7}$ power-law measured for comet Halley ((Tokunaga et al. 1992), (Waniak 1992)). Thus if Comet Shoemaker-Levy 9 had produced small grains in the same proportions as Comet Halley then they would have been well above detection limits, as indicated by Fig. 3. It is possible that this overabundance of large grains in the S-L 9 comae is due to its tidally disrupted nature - since any ancient surface mantle is largely absent, large dust grains might escape the fragments with greater ease.

The dust outflow velocity distribution $V(R)$ is given in Fig. 4 for the detected $10 \mu \mathrm{m} \leq R \leq$ $3 \mathrm{~mm}$ grains, and obeys a power-law relation $V \propto R^{-b}$ having $b=0.3 \pm 0.1$. The observed powerlaw dependence is somewhat weaker than $b=0.5$ predicted by the theory of dusty-gas emission from cometary surfaces ((Gombosi et al. 1986)), but this finding is typical of other comet studies (e.g., (Fulle 1990), (Fulle 1992), (Fulle 1996); (Waniak 1992)). The observed velocities are also in good agreement with earlier estimates ((Hahn et al. 1996), (Rettig et al. 1996)).

The upper limits on the production rates reported in Fig. 3 for grains $R \leq 3 \mu \mathrm{m}$ assume a dust outflow velocity of $3.5 \mathrm{~m} / \mathrm{sec}$. However if the small grains were ejected at a different velocity $V^{\prime}$, then the upper limits $d \dot{N}$ and $d \dot{M}$ for these grains should be adjusted by a factor $V^{\prime} /(3.5 \mathrm{~m} / \mathrm{sec})$ since imaging can only constrain the grains' column density which varies as $d \dot{N}(R) / V(R)$. The upper limit on the production of $R=1 \mathrm{~cm}$ grains assumes an outflow velocity $V^{\prime}=1 \mathrm{~m} / \mathrm{sec}$ (the arrow in Fig. 4) and thus yields a conservative limit on the mass loss rate for these grains.

The statistical uncertainties quoted in the Figs. 3-4 represent $68 \%$ confidence intervals in the model parameters assuming a constant dust grain density $\rho_{d}=1 \mathrm{gm} / \mathrm{cm}^{3}$ and a dust albedo $a=0.04$. Thus the results reported here are further affected by the systematic uncertainties associated with the unknown bulk density of comet dust and their albedo. Radiation pressure sorts dust grains according to the product $\rho_{d} R$, so if the true S-L 9 grain density $\rho_{d}$ differs from the value assumed here then the $R$ axis in Figs. 3-4 should be divided by a factor $\rho_{d}$ expressed in cgs units. The observed flux reflected by each grain size bin determines $a R^{2} d \dot{N}(R)$, so if an alternate albedo $a$ is also preferred, the grain production rates in Fig. 3 should be multiplied by $0.04 \rho_{d}^{2} / a$. However if the grain density $\rho_{d}$ is independent of grain size $R$ (which might not be true if the smaller cometary grains are fluffy instead of compact), then the mass loss rates $d \dot{M}(R) \propto \rho_{d} R^{3} d \dot{N}(R)$ reported in Fig. 3 are independent of the assumed grain density but still uncertain by a factor of $0.04 / a$.

The parameter search yields a phase law coefficient $\beta=0.024 \pm 0.003$ magnitudes/degree, which is typical for comets that generally exhibit $\beta=0.01$ to $0.04 \mathrm{mag} /$ degree ((Meech and Jewitt 1987)). The colors of the S-L 9 comae were also similar to most other comets ((Cochran et al. 1994), 
(Meech and Weaver 1996)), further indicating that the light scattering properties of the S-L 9 dust were otherwise unremarkable.

Figures 5-10 show the model fits for fragments H, K, and L as well as their dust size/velocity distributions (results for fragment $\mathrm{K}$ are also reported in (Rettig and Hahn 1997)). All the important characteristics extracted from these fits are summarized in Table II which lists the dust mass loss rate $\dot{M}$ for the detected grains, the power-law indices $a$ and $b$ for the dust size/velocity distributions, the upper limit on the fragment radius $R_{\mathrm{f}, \max }$, and the dust phase coefficient $\beta$. In general the model is able to reproduce all the features exhibited by the S-L 9 tails, such as their westward orientation seen just after solar opposition as well as the comae elongation and enhanced brightness toward Jupiter that was observed as the fragments approached the planet. However systematic discrepancies are evident, for the simulated isophotes very often lie just interior to the observed tailward isophotes. Better agreement might be achieved with a model that considers anisotropic or perhaps spherical dust emission patterns rather than the hemispherical dust emission considered here. Also recall that the dust tail model is time-independent, so the dust production rates reported here should be considered an average over the six-month span of S-L 9 observations considered here. Better agreement between the simulations and observations might also be achieved with a time-dependent model of dust production.

\subsection{Fragments $\mathrm{S}$ and $\mathrm{T}$}

The parameter search algorithm was unable to find a satisfactory fit to the observations of fragment S, the contours of which are shown in Fig. 11. This failure is most likely a consequence of a bilateral asymmetry evident in this fragment's coma and tail that was first described as a "brightness spur" ((Weaver 1994a), (Weaver 1994b)). This asymmetry is revealed in Fig. 12 which shows the ratio of the surface brightness of fragment $\mathrm{S}$ to the average of fragments $\mathrm{G}$, H, and K's surface brightness. The white regions in Fig. 12 indicate where fragment S's coma and tail dust was brighter than the averaged fragments' dust by at least a factor of 1.8. The feature extends south from the coma photocenter and trails westward, presumably due to radiation pressure, along the southern edge of the dust tail and in the general direction of fragment $\mathrm{T}$ (the white patch to the west in Fig. 12). Inspection of subsequent observations of fragment $\mathrm{S}$ shows that the morphology and orientation of the dust stream was stationary over several months. Although it had the same appearance in March, by May the feature was less distinct and by June it had largely dissipated.

Since the orientation of the dust stream did not vary with time, it is unlikely that the dust stream formed from dust jetting from an active spot on the fragment's surface unless the fragment had a very long rotation period $\gg 3$ months or the jet lie near the rotation axis. It instead seems more likely that the fragment experienced a brief outburst of material from a localized spot on the fragment's surface. Radiation pressure would have subsequently deflected the cloud of dust in the anti-sunward direction, and, owing to a dispersion in grain sizes, elongated it into the dust stream as seen in the processed image (Fig. 12). Close inspection to the contour maps (Fig. 11) shows that 
this dust stream was also evident in the unprocessed images.

The proximity of the dust stream to the coma of fragment $\mathrm{T}$ might suggest that they were created during the same outburst. However this explanation is problematic. In such a scenario, "fragment" $\mathrm{T}$ must have been a cloud of debris consisting of sub-centimeter grains that were driven tailward by radiation pressure (this is not unreasonable considering that $\mathrm{T}$ diminished in brightness over time and did not exhibit an impact signature ((Hammel et al. 1995), (Chodas and Yeomans 1996))). But if T's coma consisted of the smallest and hence fastest grains that led the dust cloud downstream, then a seemingly ad hoc gap in the dust size distribution is required to account for the spatial gap observed between the end of the dust stream and fragment T (Fig. 12). One can also extrapolate the dust stream's seemingly parabolic path down the tail as projected onto the sky plane. However the extrapolation does not contact fragment T's coma, further suggesting separate origins for the two objects.

Fragments G, H, K, and L were also examined by constructing ratios of their imaged surface brightnesses to the average of the remaining fragments (excepting L and S), and none revealed any substructure in their dust comae and tails.

\subsection{Fragment Size Estimates}

The image-fitting algorithm did not detect any fragments that were sufficiently large such that their light contribution could be distinguished from their comae light and the photon counting noise. Thus only upper limits have been established for the fragments' radii, i.e., $R_{\mathrm{f}, \max } \simeq 1.0-1.5$ $\mathrm{km}$ assuming an albedo $a=0.04$ (Table II). These upper limits are robust. As a test of the algorithm, the light contributed by an unresolved light source of radius $1.5 \times R_{\mathrm{f} \text {, max }}$ is coadded to all of the S-L 9 images and aligned with their comae photocenters. Rerunning the parameter search routine returns the expected fragment radius of $1.5 R_{\mathrm{f}}$ max with an uncertainty of $\sim 25 \%$. We also note that these dust comae have optical depths of $\tau \sim(2$ to 4$) \times 10^{-5}$ in a $0.3^{\prime \prime}$-wide box centered on the comae photocenters. Thus if these fragments have sizes near their upper limits, their additional light-contribution would be at the $\sim 10$ to $20 \%$ level. Note also that the size limits obtained for fragments $\mathrm{H}$ and $\mathrm{K}$ are comparable to the fragment sizes reported as detections by Sekanina ((Sekanina 1995), (Sekanina 1996)), and that the upper limit reported for fragment G

in Table II is significantly smaller than Sekanina's $R_{f} \simeq 2 \mathrm{~km}$ measurement. However, solving the Humpty-Dumpty problem using Sekanina's (1995) and (1996) fragment size estimates would require a progenitor comet having a radius of at least $7 \mathrm{~km}$, which is considerably larger than most other size estimates reported elsewhere.

The gravitating 'rubble-pile' variety of tidal breakup models favor an S-L 9 progenitor radius of 0.75-0.9 km ((Asphaug and Benz 1994), (Asphaug and Benz 1996), (Solem 1994)). If one assumes the tidally disrupted S-L 9 debris was mostly contained among the 10 or so bright on-axis fragments, then their average radii was $\sim 0.4 \mathrm{~km}$. Indeed, models of the fragments' atmospheric 
entry and the subsequent fireball development indicate the impactors had radii $R_{f} \lesssim 0.25$ to $0.5 \mathrm{~km}$ ((Mac Low 1996), (Molina et al. 1997); however see (Sekanina 1996)). However some of the fragments might have been larger as suggested by the amount of post-impact debris seen in Jupiter's atmosphere. Millimeter observations of $\mathrm{CO}$ emission at the impact sites of fragments $\mathrm{G}$ and $\mathrm{K}$ yield a CO mass estimate of $\sim 10^{14-15}$ gm ((Lellouch 1996), (Lellouch et al. 1997)). While the jovian atmosphere may have contributed some if not most of the observed carbon via impactinduced chemistry, the oxygen was probably of cometary origin since recent measurements indicate Jupiter's atmosphere is deficient in oxygen ((Niemann et al. 1996), (Encrenaz et al. 1996)). These CO measurements thus place a lower limit of $R_{f} \gtrsim 0.35 \mathrm{~km}$ on the radii of fragments $\mathrm{G}$ and $\mathrm{K}$.

However the S-L 9 fragments could not have been very much smaller than the limit $R_{f, \max }$ established here, for otherwise their vigorous dust emission would result in complete evaporation before striking Jupiter. The lower limit on their radii is $R_{f, \text { min }}=\left(3 \dot{M} \Delta t / 4 \pi \rho_{f}\right)^{1 / 3} \sim 100$ meters in order for a fragment of density $\rho_{f}=0.6 \mathrm{gm} / \mathrm{cm}^{3}$ to sustain a mass loss rate of $\dot{M} \sim 20 \mathrm{~kg} / \mathrm{sec}$ during the $\Delta t \simeq 2$ year passage about Jupiter. Consequently, these observations constrain the radii of the these fragments to lie within the size interval $0.1 \lesssim R_{f} \lesssim 1.5 \mathrm{~km}$, without these fragments ever actually having been observed directly. The fragment size limits obtained here are in good agreement with models of the tidal breakup event as well as observations and models of the Jupiter impacts, which suggest the S-L 9 impactors had radii $R_{f} \sim$ a few hundred meters. But due to a rather vigorous production of dust, some of these fragments may have lost appreciable amounts of mass throughout their orbit. Note that fragments $\mathrm{F}, \mathrm{J}, \mathrm{P}_{1}, \mathrm{P}_{2}, \mathrm{~T}$, and $\mathrm{U}$ did not exhibit impact signatures ((Hammel et al. 1995), (Chodas and Yeomans 1996)), and these bodies may simply have exhausted most of their mass prior to impact.

\section{Summary and Conclusions}

The dust size and velocity distributions for Comet Shoemaker-Levy 9 fragments G, H, K, and L have been extracted from Hubble Space Telescope observations by fitting a model of a cometary dust tail to the data. It is assumed here that the S-L 9 dust production was steady since the moment of tidal breakup, that dust emission was isotropic across each fragment's sunlit hemisphere, and that the dust grains had an albedo $=0.04$ and bulk density $\rho_{d}=1 \mathrm{gm} / \mathrm{cm}^{3}$ independent of grain size. Since radiation pressure spatially segregates dust according to size, image-fitting techniques yield production rates for the grains of radii $10 \mu \mathrm{m} \lesssim R \lesssim 3 \mathrm{~mm}$ that dominate the dust comae optical depth. Upper limits are established for the production of grains outside this size window. The S-L 9 grains studied here are considerably larger than the $\sim 1-10 \mu \mathrm{m}$ grains that usually dominate the scattering of sunlight by most cometary comae ((Fulle 1987), (Grün and Jessberger 1990)). The S-L 9 dust size distribution power-law size index, which averages to $a=2.3 \pm 0.1$ for the fragments studied here, is considerably shallower than the $a \simeq 3$ to 4 reported for many other comets (e.g., (Tokunaga et al. 1986), (Waniak 1992), (Fulle 1992), (Fulle 1994), (Sekanina et al. 1992)). The average phase coefficient for these four fragments is $\beta=0.018 \pm 0.003$ magnitudes/degree. 
The dust tail model employed here failed to find a satisfactory fit to images of fragment $\mathrm{S}$ due to an asymmetric dust feature present in this fragment's coma. As this feature appears stationary for at least 3 months, it is more likely the remnant of a brief outburst of dust (rather than a steady jet) from a localized spot on the fragment's surface, with the burst being activated prior to detection on January 25, 1994. The comae of the remaining fragments were otherwise featureless.

The coma fitting algorithm could not uniquely disentangle the sunlight reflected by a spatially distributed dust coma from the sunlight reflected by an unresolved comet fragment; this yields only upper limits on the radii of the fragments, $R_{\mathrm{f}, \max } \simeq 1.0-1.5 \mathrm{~km}$ assuming an albedo $=0.04$. But to sustain the rather heavy dust production rate of $\dot{M} \sim 20 \mathrm{~kg} / \mathrm{sec}$ during their final two year orbit about Jupiter, these fragments must have had radii in excess of $\sim 0.1 \mathrm{~km}$. This vigorous dust production might have been a consequence of tidal disruption, which effectively removed any ancient surface mantle from the S-L 9 parent body that would otherwise inhibit the emission of large dust grains. It is also noted that several other S-L 9 fragments disappeared from view without leaving any evidence of an impact with Jupiter. One possible explanation for this phenomenon is that these bodies had small nuclei that simply evaporated en route due to a vigorous production of dust.

\section{ACKNOWLEDGMENTS}

The authors that Casey Lisse for his careful review of this paper. Support for this work was provided by NASA through grant 5624.21-93A from the Space Telescope Science Institute, which is operated by the Association of Universities for Research in Astronomy, Incorporated, under NASA contract NAS5-26555. This paper is contribution XXX from the Lunar and Planetary Institute, which is operated by the Universities Space Research Association under NASA contract NASW4574 . 


\section{REFERENCES}

Asphaug, E., and W. Benz 1994. Density of Comet Shoemaker-Levy 9 deduced by modeling breakup of the parent 'rubble pile'. Nature 370, 120-123.

Asphaug, E., and W. Benz 1996. Size, density, and structure of Comet Shoemaker-Levy 9 inferred from the physics of tidal breakup. Icarus 121, 225-248.

Biver, N., H. Rauer, D. Despois, R. Moreno, G. Paubert, D. Bockelée-Morvan, P. Colom, J. Crovisier, E. Gérard, and L. Jorda 1996. Substantial outgassing of CO from comet HaleBopp at large heliocentric distance. Nature 380, 137-139.

Burns, J. A., P. L. Lamy, and S. Soter 1979. Radiation forces on small particles in the solar system. Icarus 40, 1-48.

Chodas, P. W., and D. K. Yeomans 1996. The orbital motion and impact circumstances of Comet Shoemaker-Levy 9. In The Collision of Comet Shoemaker-Levy 9 and Jupiter (K. S. Noll, H. A. Weaver, and P. D. Feldman, Eds.), pp. 1-30. IAU Colloquium 156.

Cochran, A. L., A. L. Whipple, P. J. MacQueen, P. J. Shelus, R. W. Whited, and C. F. Claver, 1994. Preimpact characterization of $\mathrm{P} /$ Comet Shoemaker-Levy 9. Icarus 112, 528-532.

Encrenaz, Th., and 19 colleagues, 1996. First results of ISO-SWS observations of Jupiter. Astron. Astrophys 315, L397-L400.

Fulle, M. 1987. A new approach to the Finson-Probstein method of interpreting cometary dust tails. Astron. Astrophys 171, 327-335.

Fulle, M. 1990. Meteoroids from short period comets. Astron. Astrophys 230, 220-226.

Fulle, M. 1992. Dust from short period comet P/Schwassmann-Wachmann 1 and replenishment of the interplanetary dust cloud. Nature 359, 42-44.

Fulle, M. 1994. Spin axis orientation of 2060 Chiron from dust coma modeling. Astron. Astrophys 282, 980-988.

Fulle, M. 1996. Dust environment and nucleus spin axis of comet P/Tempel 2 from models of the infrared dust tail observed by IRAS. Astron. Astrophys. 311, 333-339.

Grün, E., and E. Jessberger 1990. Dust. In Physics and Chemistry of Comets (W. F. Huebner, Ed.), pp. 113-175. Springer-Verlag, Berlin.

Gombosi, T. I., A. F. Nagy, and T. E. Cravens 1986. Dust and neutral gas modeling of the inner atmospheres of comets. Rev. Geophys. 24, 667-700.

Hahn, J. M., T. W. Rettig, and M. J. Mumma 1996. Comet Shoemaker-Levy 9 dust. Icarus 121, 291-304. 
Hammel, H. B., R. F. Beebe, A. P. Ingersoll, G.§. Orton, J. R. Mills, A. A. Simon, P. Chodas, J. T. Clarke, E. De Jong, T. E. Dowling, J. Harrington, L. F. Huber, E. Karkoschka, C. M. Santori, A. Toigo, D. Yeomans, and R. A. West 1995. HST imaging of atmospheric phenomena created by the impact of Comet Shoemaker-Levy 9. Science 267, 1288-1296.

Hanner, M. S., and R. L. Newburn 1989. Infrared photometry of Comet Wilson (19861) at two epochs. AJ 97, 254-261.

Krist, J. 1994. The Tiny Tim User's Manual. STScI Publication.

Lellouch, L. 1996. Chemistry induced by impacts: Observations. In The Collision of Comet Shoemaker-Levy 9 and Jupiter, (K. S. Noll, H. A. Weaver, and P. D. Feldman, Eds.), pp. 213-242. IAU Colloquium 156.

Lellouch, E., B. Bézard, R. Moreno, D. Bocklelée-Morvan, P. Colom, J. Crovisier, M. Festou, D. Gautier, A. Marten, and G. Paubert 1997. Carbon monoxide in Jupiter after the impact of comet Shoemaker-Levy 9. Planet. and Space Sci. 45, 1203-1212.

Lester, T. P., M. L. McCall, and J. B. Tatum 1979. Theory of Planetary Photometry. J. Roy. Astron. Soc. Can. 73, 233-257.

Lorenzetti, D., A. Monetti, R. Stanga, and F. Strafella 1987. Infrared monitoring of comet P/Halley. Astron. Astrophys 187, 609-615.

Lux, I., and L. Koblinger 1991. Monte Carlo Particle Transport Methods: Neutron and Photon Calculations, CRC Press, Boston.

Mac Low, M.-M. 1996. Entry and fireball modes vs. observations: What have we learned? In The Collision of Comet Shoemaker-Levy 9 and Jupiter, (K. S. Noll, H. A. Weaver, and P. D. Feldman, Eds.), pp. 157-182. IAU Colloquium 156.

Meech, K. J., and D. C. Jewitt 1987. Observations of comet P/Halley at minimum phase angle. Astron. Astrophys. 187, 585-593.

Meech, K. J., M. J. S. Belton, B. E. A. Mueller, M. W. Dickison, and H. R. Li 1993. Nucleus properties of $\mathrm{P} /$ Schwassmann-Wachmann 1. AJ 106, 1222-1236.

Meech, K. J., and H. A. Weaver 1996. Unusual comets (?) as observed from the Hubble Space Telescope. Earth, Moon, and Planets 72, 119-132.

Molina, A., F. Moreno, and O. Muñoz 1997. A discussion on impact models of the Shoemaker-Levy 9 fragments with Jupiter from some observational results. Planet. Space Sci. 45, 1279-1285.

Nelder, J. A., and R. Mead 1965. A simplex method for function minimization. Computer Journal 7, 308-313. 
Niemann, H. B., S. K. Atreya, G. R. Carignan, T. M. Donahue, J. A. Haberman, D. N. Harpold, R. E. Hartle, D. M. Hunten, W. T. Kasprzak, P. R. Mahaffy, T. C. Owen, N. W. Spencer, S. H. Way 1996. The Galileo probe mass spectrometer: Composition of Jupiter's atmosphere. Science 272, 846-848.

Parkinson, J. M., and D. Hutchinson 1972. An investigation in the efficiency of variants on the simplex method. In Numerical Methods for Non-Linear Optimization (F. A. Lootsma, Ed.) p. $115-135$.

Press, W. H., B. B. Flannery, S.A. Teukolsky, and W. T. Vetterling 1992. Numerical Recipes in C. Cambridge Univ. Press, Cambridge.

Rettig, T. W., G. W. Sobczak, and J. M. Hahn 1996. Dust outflow velocity for Comet ShoemakerLevy 9. Icarus 121, 281-290.

Rettig, T. W., and J. M. Hahn 1997. Comet Shoemaker-Levy 9: An active comet. Planet. Space Sci. 45, 1271-1277.

Sagdeev, R. Z., G. A. Avanesov, Y. L. Ziman, V. I. Moroz, V. I. Tarnopolsky, B. S. Zhukov, and V. A. Shamis 1986. TV experiment of the Vega mission: Photometry of the nucleus and the inner coma. In Exploration of Halley's Comet (B. Battrick, E. J. Rolfe, and R. Reinhard, Eds.), ESA SP-250, pp. 317-326.

Sekanina, Z. 1995. Evidence on sizes and fragmentation of the nuclei of Comet Shoemaker-Levy 9 from Hubble Space Telescope images. Astron. Astrophys. 304, 296-316.

Sekanina, Z. 1996. Fragmentation of Comet Shoemaker-Levy 9's nuclei during flight through the jovian atmosphere. In Physics, Chemistry, and Dynamics of Interplanetary Dust, (B. A. S. Gustafson, and M. S. Hanner, Eds.), pp. 383-386. ASP Conference Series 104.

Sekanina, Z., S. M. Larson, O. Hainaut, A. Smette, and R. M. West 1992. Major outburst of periodic comet Halley at a heliocentric distance of 14 AU. Astron. Astrophys. 263, 367-386.

Sekanina, Z.,, P. W. Choda, and D. K. Yeomans 1994. Tidal disruption and the appearance of periodic comet Shoemaker-Levy 9. Astron. Astrophys. 289, 607-636.

Senay, M. C. and D. Jewitt 1994. Coma formation driven by carbon monoxide release from comet Schwassmann-Wachmann 1. Nature, 371, 229-231.

Solem, J. C. 1994. Density and Size of Comet Shoemaker-Levy 9 deduced from a tidal breakup model. Nature 370, 349-351.

Standish, E. M., X. X. Newhall, J. G. Williams, and W. M. Folkner 1995. JPL Planetary and Lunar Ephemerides, DE403/LE403. JPL IOM 314.10-127. 
Tanigawa, T., H. Kawakita, and J. Watanabe 1997. The activity of the fragmented nucleus of comet Shoemaker-Levy 9. Planet. Space Sci. 45, 1417-1422.

Tokunaga, A. T., W. F. Golisch, D. M. Griep, C. D. Kaminski, and M. S. Hanner 1986. The NASA Infrared Telescope Facility Comet Halley monitoring program. I. Preperihelion results. Astron. J. 92, 1183-1190.

Tokunaga, A. T., M. S. Hanner, W. F. Golisch, D. M. Griep, C. D. Kaminski, and H. Chen 1992. Infrared monitoring of Comet $\mathrm{P} /$ Temple 2. Astron. J. 104, 1611-1617.

Waniak, W. 1992. A Monte Carlo approach to the analysis of the dust tail of Comet P/Halley. Icarus 100, 154-161.

Weaver, H. A. 1994a. Periodic Comet Shoemaker-Levy 9 (1993e). IAU Circular 5947.

Weaver, H. A. 1994b. Periodic Comet Shoemaker-Levy 9 (1993e). IAU Circular 5973.

Weaver, H. A., P. D. Feldman, M. F. A’Hearn, C. Arpigny, R. A. Brown, E. F. Helin, D. H. Levy, B. G. Marsden, K. J. Meech, S. M. Larson, K. S. Noll, J. V. Scotti, Z. Sekanina, C. S. Shoemaker, E. M. Shoemaker, T. E. Smith, A. D. Storrs, D. K. Yeomans, and B. Zellner 1994. Hubble Space Telescope Observations of Comet P/Shoemaker-Levy 9 (1993e). Science 263, 787-791, 1994.

Weaver, H. A., M. F. A. A'Hearn, C. Arpigny, D. C. Boice, P. D. Feldman, S. M. Larson, P. Lamy, D. H. Levy, B. G. Marsden, K. J. Meech, K. S. Noll, J. V. Scotti, Z. Sekanina, C. S. Shoemaker, E. M. Shoemaker, T. E. Smith, S. A. Stern, A. D. Storrs, J. T. Trauger, D. K. Yeomans, and B. Zellner 1995. Hubble Space Telescope (HST) observing campaign on Comet Shoemaker-Levy 9. Science 267, 1282-1287.

Waever, H. A. and P. L. Lamy 1997. Estimating the size of Hale-Bopp's nucleus. Earth, Moon and Planets 79, 17-33.

Weissman, P. R., M. F. A'Hearn, and L. A. McFadden 1989. Evolution of comets into asteroids. In Asteroids II (R. P. Binzel, T. Gehrels, and M. S. Matthews, Eds.), pp. 880-920. Univ. of Arizona Press, Tuscon.

Weissman, P. R. 1996. If it quacks like a comet...Icarus 121, 275-280. 
Fig. 1. Contours of column density maps for an $R_{i}=300 \mu \mathrm{m}, V\left(R_{i}\right)=0.75 \mathrm{~m} / \mathrm{sec}$ model on March 31, 1994 (black curves) and June 26, 1994 (grey curves). These maps illustrate the development of dust tails due to radiation pressure as well as the influence of Jupiter's gravity as S-L 9 nears impact.

Fig. 2. Contour maps of fragment G (thick grey curves) and the dust model (thick black curves) fitted to the observations. Each successive isophote corresponds to a change in brightness by a factor of 3. The two dimmest observed isophotes are of the image smoothed over a $0.6^{\prime \prime}$ box. The 'fins' along the fainter simulated isophotes are an artifact of the model which emits dust having discrete rather than continuous sizes and velocities. The arrow in the July 14 image indicates the projected direction to Jupiter. Contours for the March fit, which appears similar to the January isophotes, are not displayed. Some isophotes appear incomplete due to rotating the images such that north is up. A star trail is responsible for the disagreement in the northwest corner of the January fit. Syndynes (narrow solid curves) are identified by their dust radius $R$, and synchrones (dashed curves) are shown for dust emitted at times $t$ prior to the observing date. In the JanuaryMay images, the smaller, fresh dust inhabit the northern side of the tail, whereas the larger, older grains reside on the southern side. In the later observations, the syndynes/synchrones segregate due to the changing viewing geometry as well as due to proximity to Jupiter.

Fig. 3. Fragment G's differential dust production rate $d \dot{N}(R)$ (read with the left axis) and mass production rate $d \dot{M}(R)$ (right axis) as a function of grain radius $R$. Arrows indicate upper limits. The dashed curve has the same logarithmic slope as an $R^{-3.7}$ Halley-type grain size distribution. These curves assume a dust albedo $a=0.04$ and a bulk density of $\rho_{d}=1 \mathrm{gm} / \mathrm{cm}^{3}$.

Fig. 4. The dust outflow velocity for fragment $\mathrm{G}$ and an $R^{-0.3}$ curve.

Fig. 5. Contour maps of fragment $\mathrm{H}$ (grey curves) and the dust model (black curves) fitted to the observations. Star trails lie south of the fragment in May, in the southwest corner in June, and north of the fragment in July.

Fig. 6. Fragment H's differential dust production rate $d \dot{N}(R)$ and mass production rate $d \dot{M}(R)$ as a function of grain radius $R$. Below is the dust outflow velocity distribution $V(R)$ as well as a $V(R) \propto R^{-b}$ curve with $b=0.5$.

Fig. 7. Contour maps of fragment K (grey curves) and the dust model (black curves) fitted to the observations. A star trail lies north of the fragment in March, the data were clipped by the detector edge in May, and this fragment was not observed on July 14.

Fig. 8. Fragment K's differential dust production rate $d \dot{N}(R)$ and mass production rate $d \dot{M}(R)$ as a function of grain radius $R$, and below a $V(R) \propto R^{-b}$ curve with $b=0.1$ is plotted over the dust 
outflow velocity distribution $V(R)$.

Fig. 9. Contour maps of fragment L (grey curves) and the dust model (black curves) fitted to the observations. Star trails lie northwest of the fragment in May and west-southwest in June.

Fig. 10. Fragment L's differential dust production rate $d \dot{N}(R)$ and mass production rate $d \dot{M}(R)$ as a function of grain radius $R$. Below is the dust outflow velocity distribution $V(R)$ as well as a $V(R) \propto R^{-b}$ curve having $b=0.3$.

Fig. 11. Contour maps of fragment $\mathrm{S}$, with fragments $\mathrm{T}$ and $\mathrm{U}$ indicated in the January image. In the March image a star trail lies at the western edge of the plot, several stars trail through the May image, and a star trail lies north of the fragment in July.

Fig. 12. The ratio of the surface brightness of fragment $\mathrm{S}$ on January 25 to the average of fragments $\mathrm{G}, \mathrm{H}$, and K, renormalized such that each image has the same integrated brightness as fragment $\mathrm{S}$. Grey areas have a surface brightness ratio of unity, and in the saturated white areas the brightness of fragment $\mathrm{S}$ exceeds the averaged fragments by more than a factor of 1.8. The bullseye indicates the coma photocenter, and the white patch to the west is fragment $\mathrm{T}$. The data become spotty as the signal diminishes with distance down the tail, and the diagram displays only those pixels where the flux ratio has a signal/noise $>1.5$. 
Table I:

TABLE I

S-L 9 OBSERVATION PARAMETERS ${ }^{a}$

\begin{tabular}{lccccc}
\hline \hline Date (1994) & $\mathrm{ID}^{b}$ & $r^{c}(\mathrm{AU})$ & $\Delta^{d}(\mathrm{AU})$ & $r_{\mathrm{J}}{ }^{e}\left(10^{6} \mathrm{~km}\right)$ & $\phi^{f}$ (degrees) \\
\hline January 25 & $\mathrm{A}-\mathrm{W}$ & 5.39 & 5.40 & 40.0 & -10.5 \\
March 31 & $\mathrm{A}-\mathrm{W}$ & 5.38 & 4.50 & 31.2 & -5.6 \\
May 18 & $\mathrm{A}-\mathrm{W}$ & 5.38 & 4.41 & 22.8 & 3.7 \\
June 26 & $\mathrm{H}$ & 5.39 & 4.80 & 12.1 & 9.5 \\
& $\mathrm{~S}$ & & & 13.2 & \\
June 27 & $\mathrm{K}$ & 5.40 & 4.82 & 12.2 & 9.5 \\
July 4 & $\mathrm{Q}_{1}$ & 5.40 & 4.91 & 10.1 & 10.0 \\
July 12 & $\mathrm{Q}_{1}$ & 5.41 & 5.03 & 6.81 & 10.5 \\
July 14 & $\mathrm{G}$ & 5.41 & 5.07 & 3.92 & 10.6 \\
& $\mathrm{H}$ & & & 4.49 & \\
July 19 & $\mathrm{S}$ & & & 5.78 & \\
July 20 & $\mathrm{K}$ & 5.42 & 5.14 & 1.37 & 10.7 \\
& $\mathrm{~L}$ & 5.41 & 5.16 & 0.86 & 10.7 \\
July 21 & $\mathrm{Q} 1$ & & & 1.01 & \\
\hline
\end{tabular}

${ }^{a}$ Provided by P.W Chodas (1995, private communication).

${ }^{b}$ Fragment ID.

${ }^{c}$ The comet's heliocentric distance.

${ }^{d}$ Geocentric distance.

${ }^{e}$ Jovicentric distance.

${ }^{f}$ Sun-comet-Earth phase angle. A minus sign indicates a pre-opposition observation. 
Table II:

TABLE II

CHARACTERISTICS OF DUST MODEL FITS TO S-L 9 FRAGMENTS ${ }^{a}$

\begin{tabular}{cccccc}
\hline \hline Fragment & $\dot{M}^{b}(\mathrm{~kg} / \mathrm{sec})$ & $a^{c}$ & $b^{d}$ & $R_{\mathrm{f}, \max }{ }^{e}(\mathrm{~km})$ & $\beta^{f}(\mathrm{mag} /$ degree $)$ \\
\hline $\mathrm{G}$ & $22 \pm 5$ & $2.2 \pm 0.1$ & $0.3 \pm 0.1$ & 1.0 & $0.024 \pm 0.003$ \\
$\mathrm{H}$ & $15 \pm 3$ & $2.4 \pm 0.2$ & $0.5 \pm 0.2$ & 1.5 & $0.030 \pm 0.005$ \\
$\mathrm{~K}$ & $22 \pm 5$ & $2.2 \pm 0.2$ & $0.1 \pm 0.2$ & 1.2 & $0.009 \pm 0.006$ \\
$\mathrm{~L}$ & $6 \pm 2$ & $2.2 \pm 0.2$ & $0.3 \pm 0.2$ & 1.1 & $0.010 \pm 0.010$ \\
\hline
\end{tabular}

${ }^{a}$ All error estimates and upper limits represent the fit's $68 \%$ confidence intervals.

${ }^{b}$ Production rates for the grains detected in the fragment's coma, i.e., for $R \lesssim 3 \mathrm{~mm}$ grains.

${ }^{c}$ Power law index for the differential dust production rate $d \dot{N}(R) \propto R^{-a}$.

${ }^{d}$ Power law index for the dust outflow velocity distribution $V(R) \propto R^{-b}$.

${ }^{e}$ Upper limit on the fragment radius.

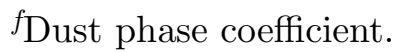




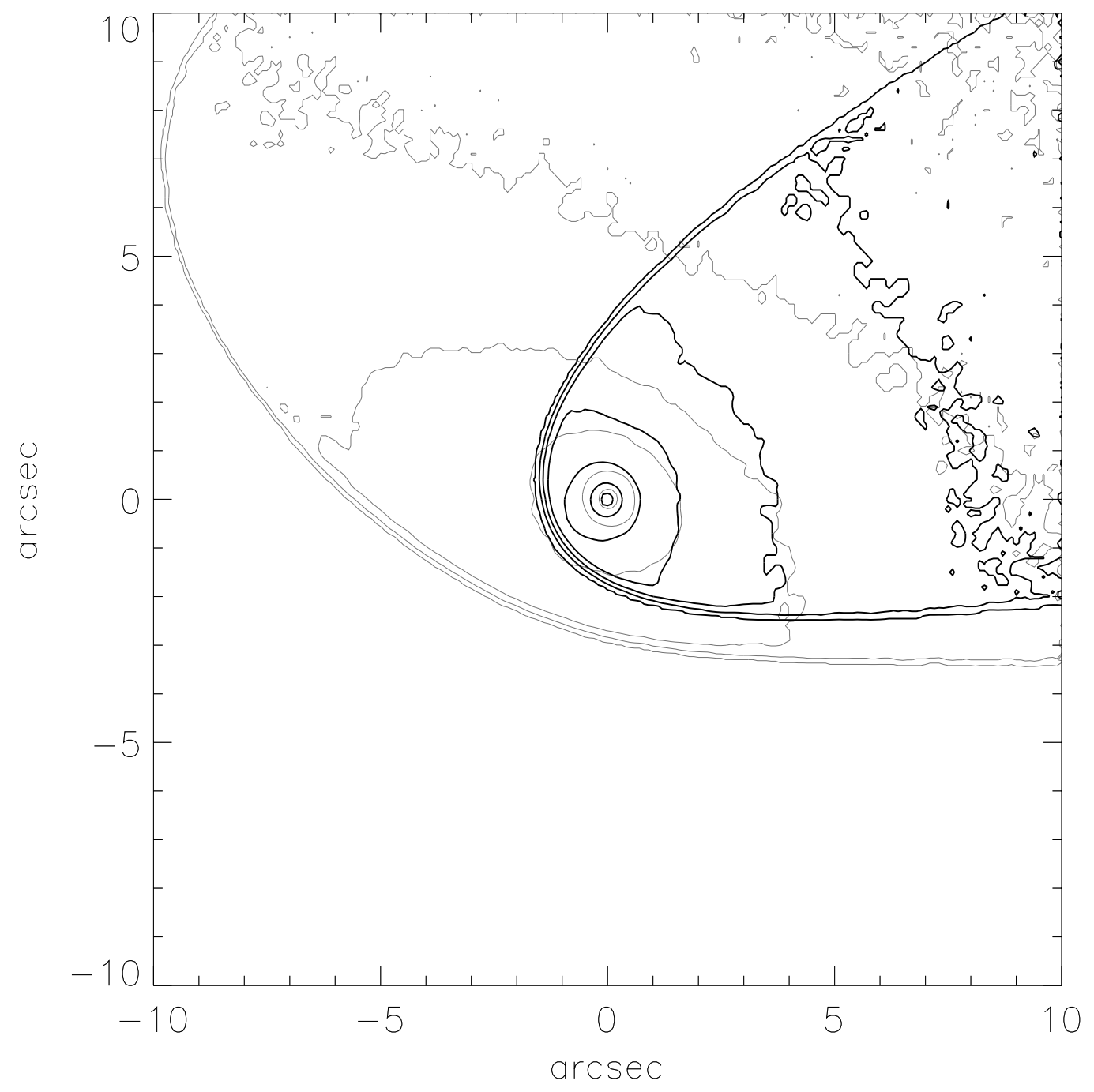

Fig. 1.- 

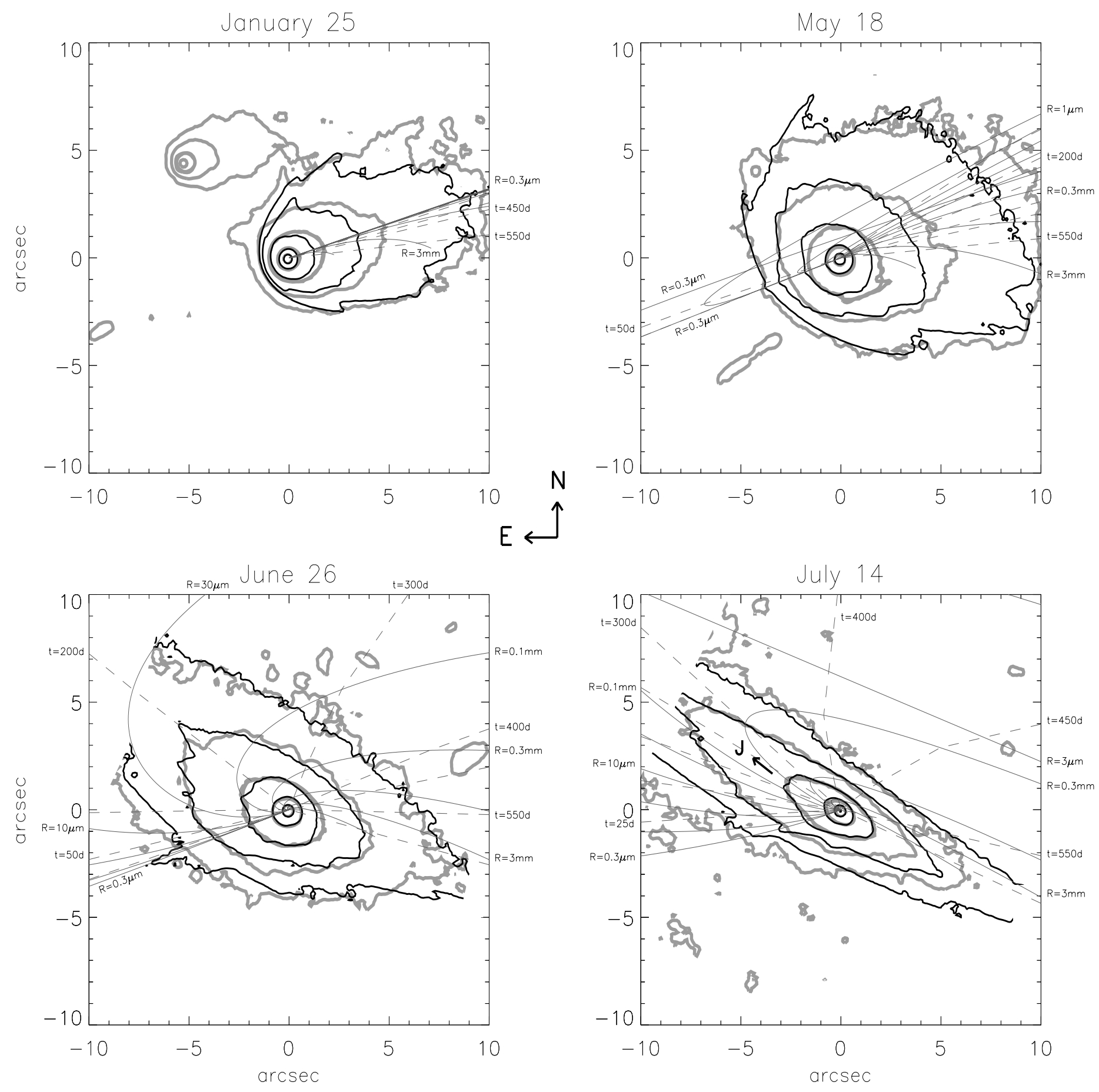

Fig. 2.- 


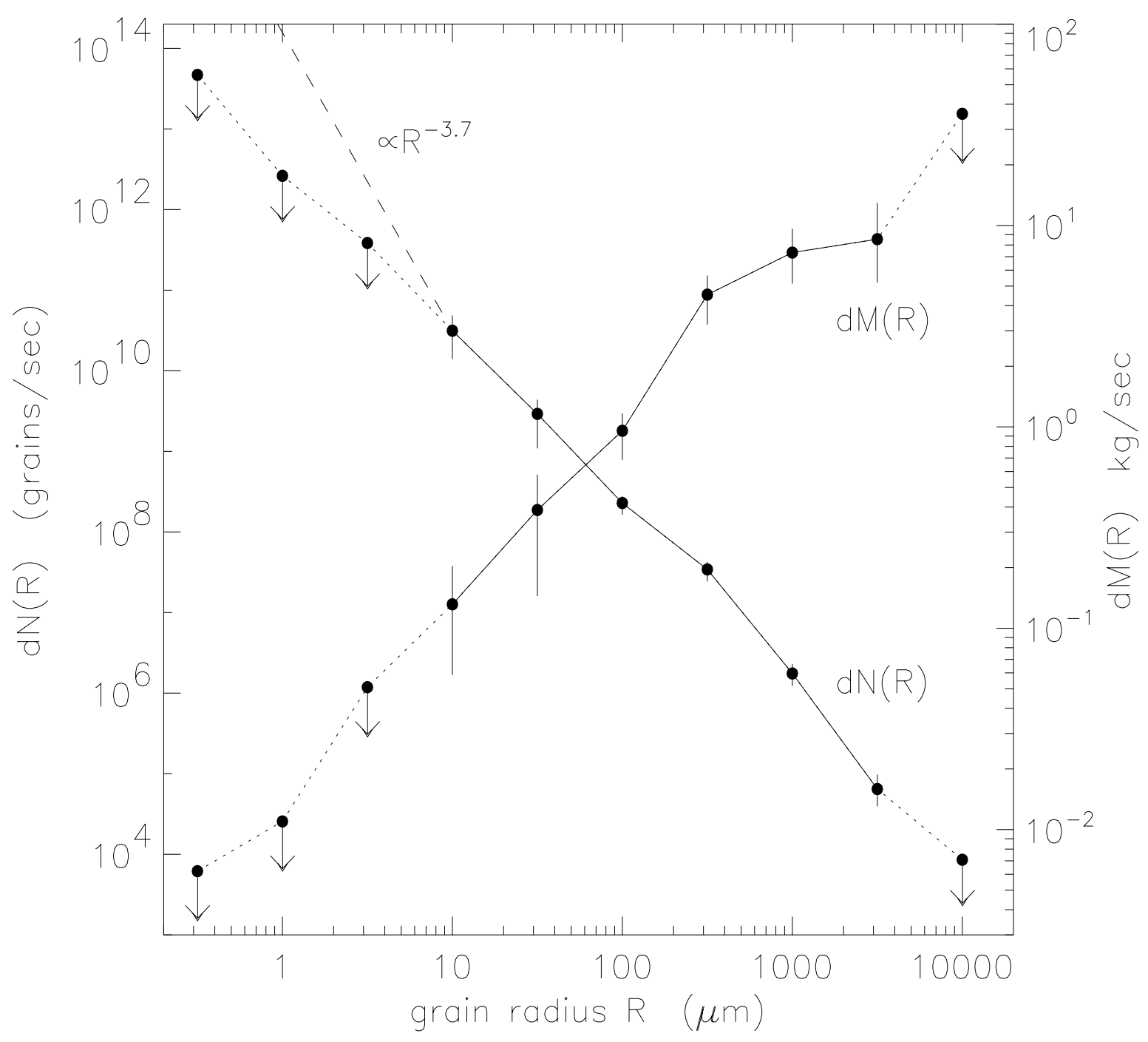

Fig. 3.- 


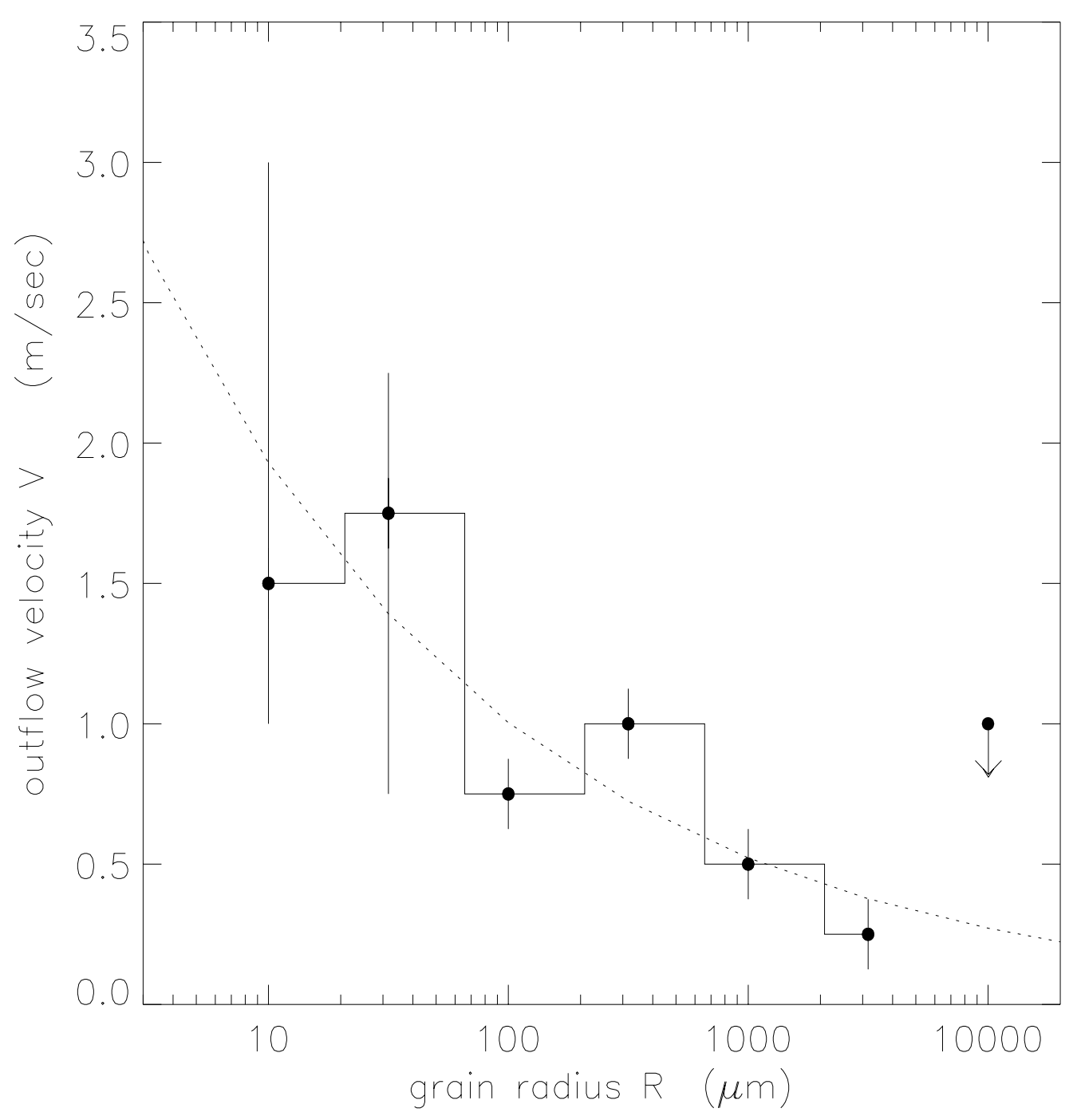

Fig. 4.- 

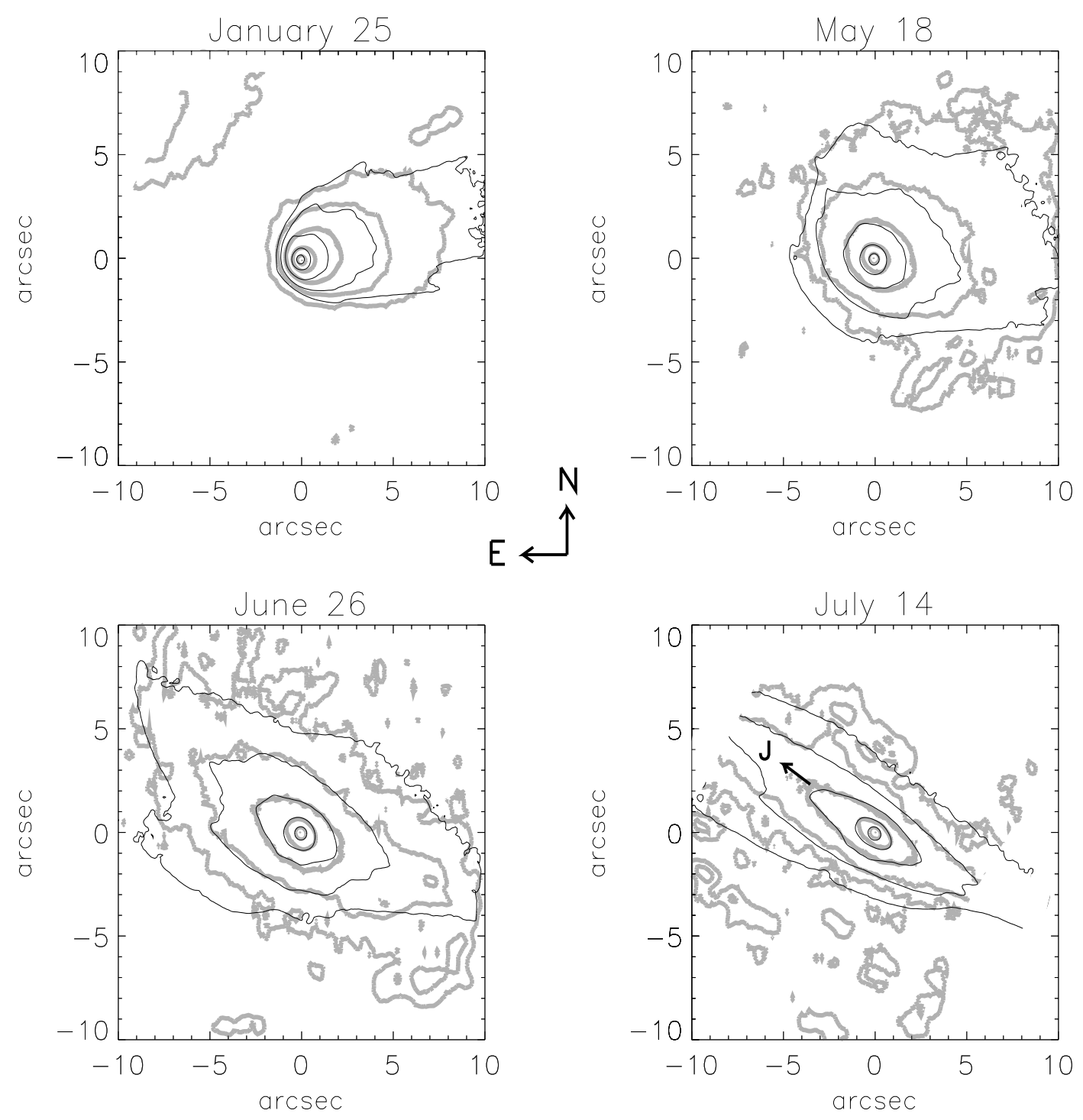

Fig. 5.- 

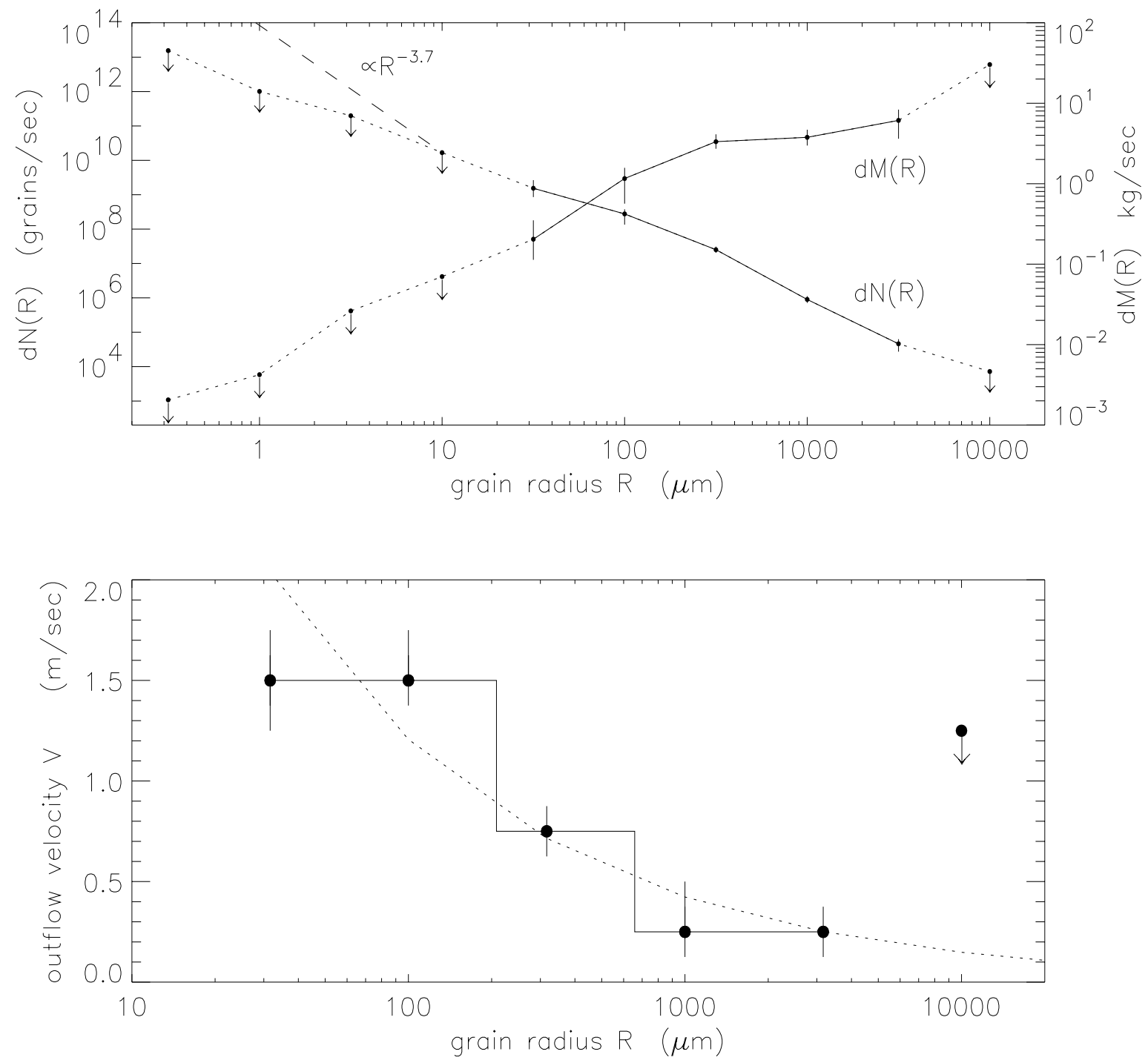

Fig. 6.- 

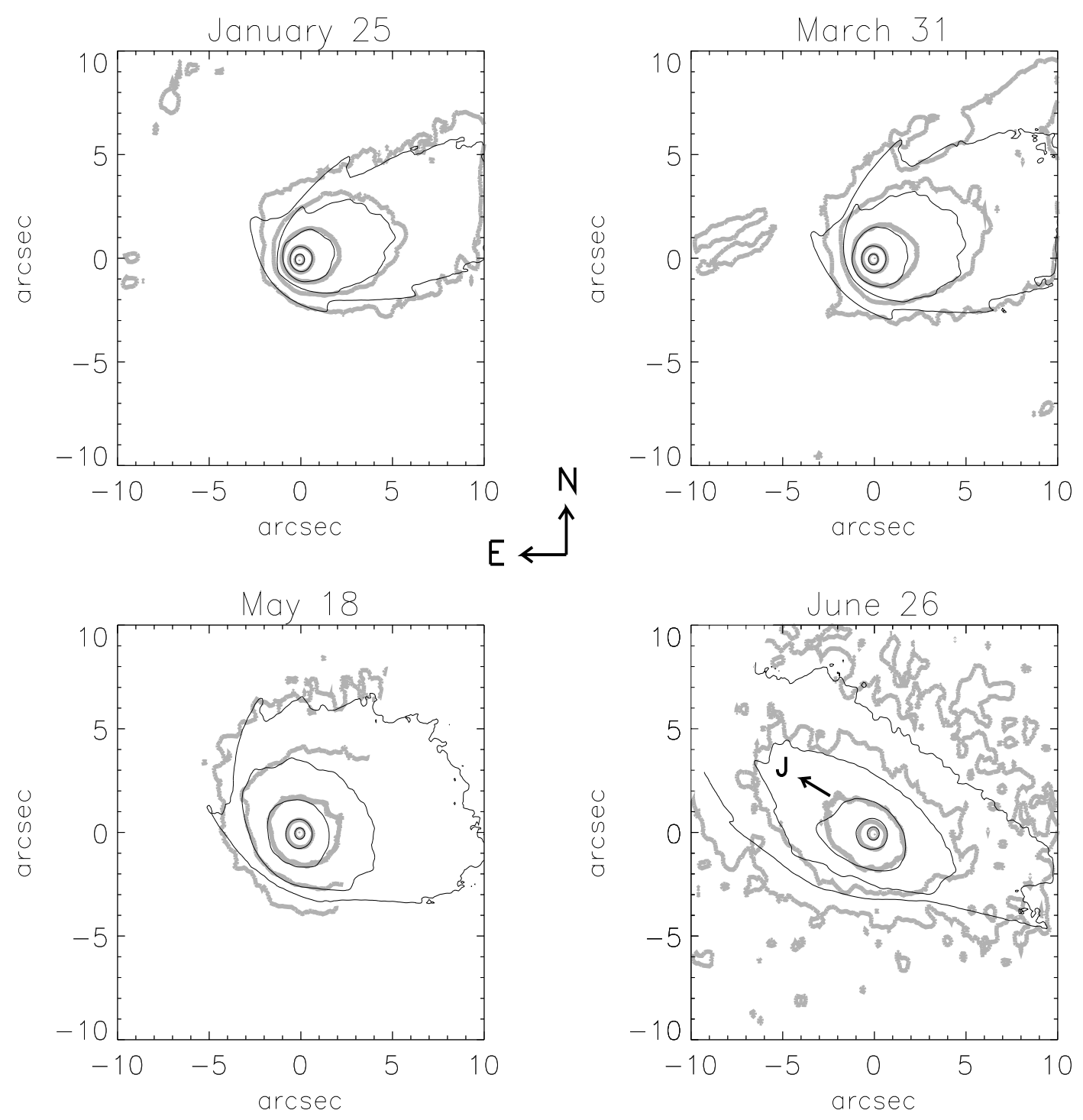

Fig. 7.- 

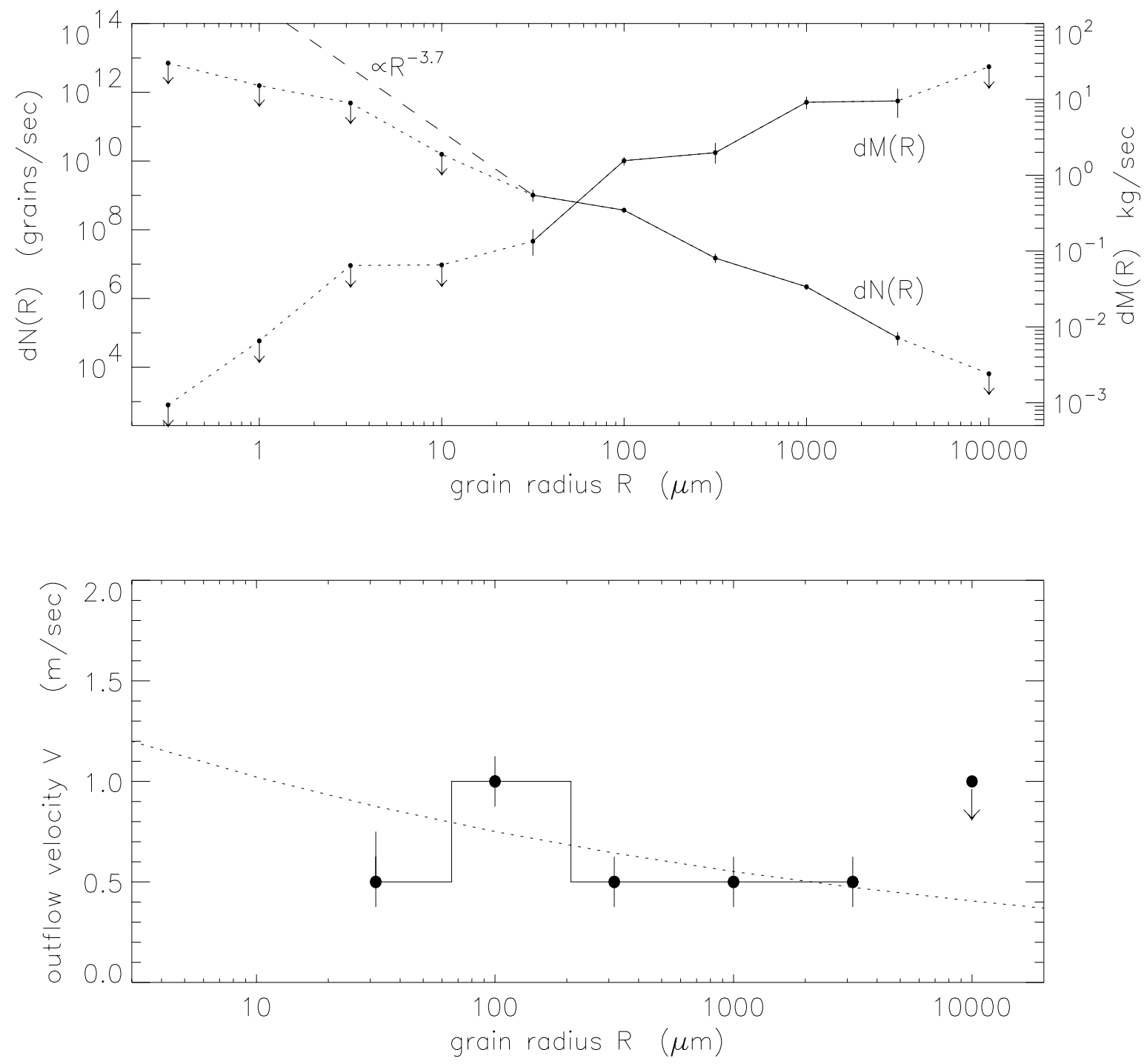

Fig. 8.- 

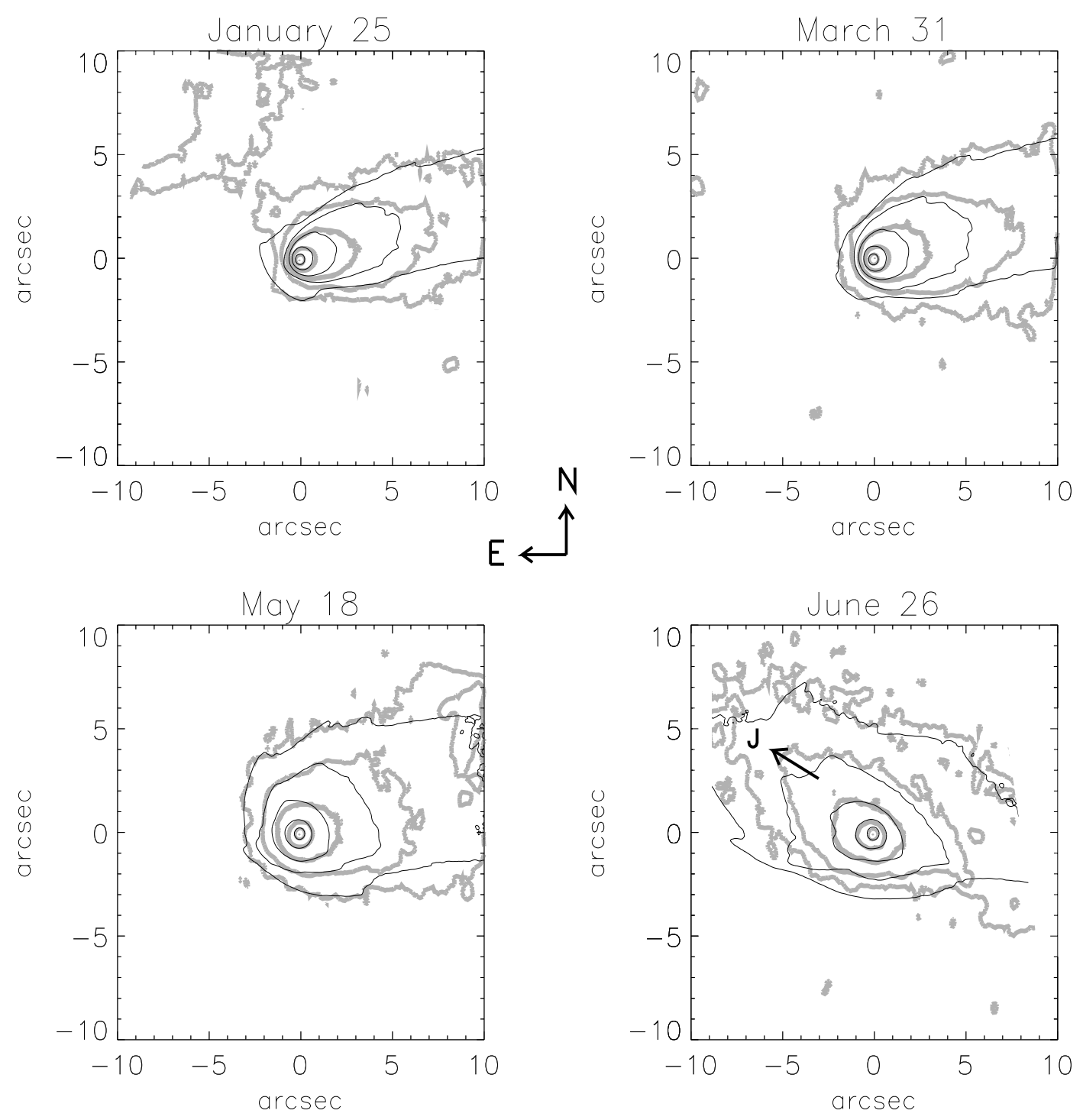

Fig. 9.- 

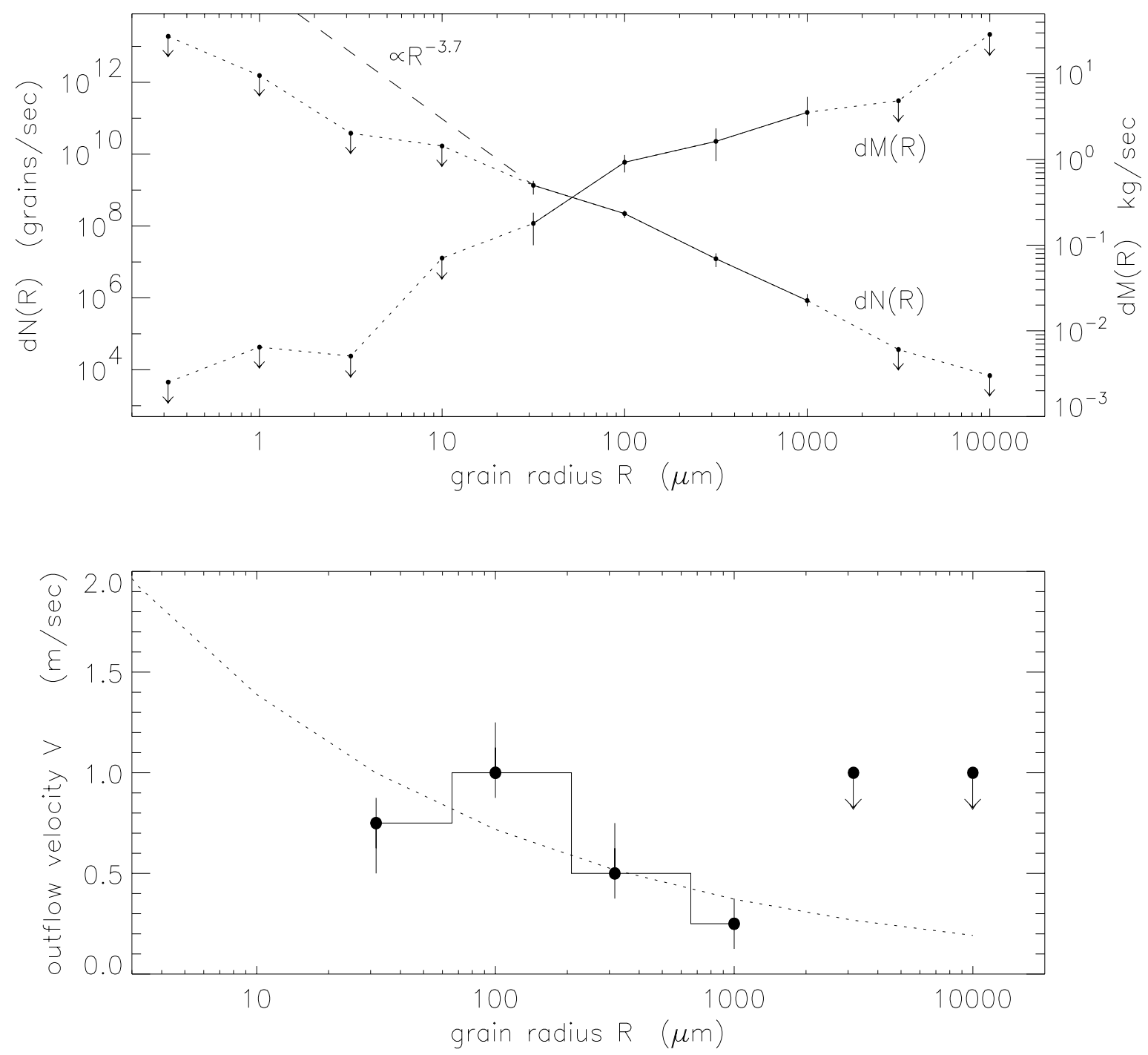

Fig. 10.- 

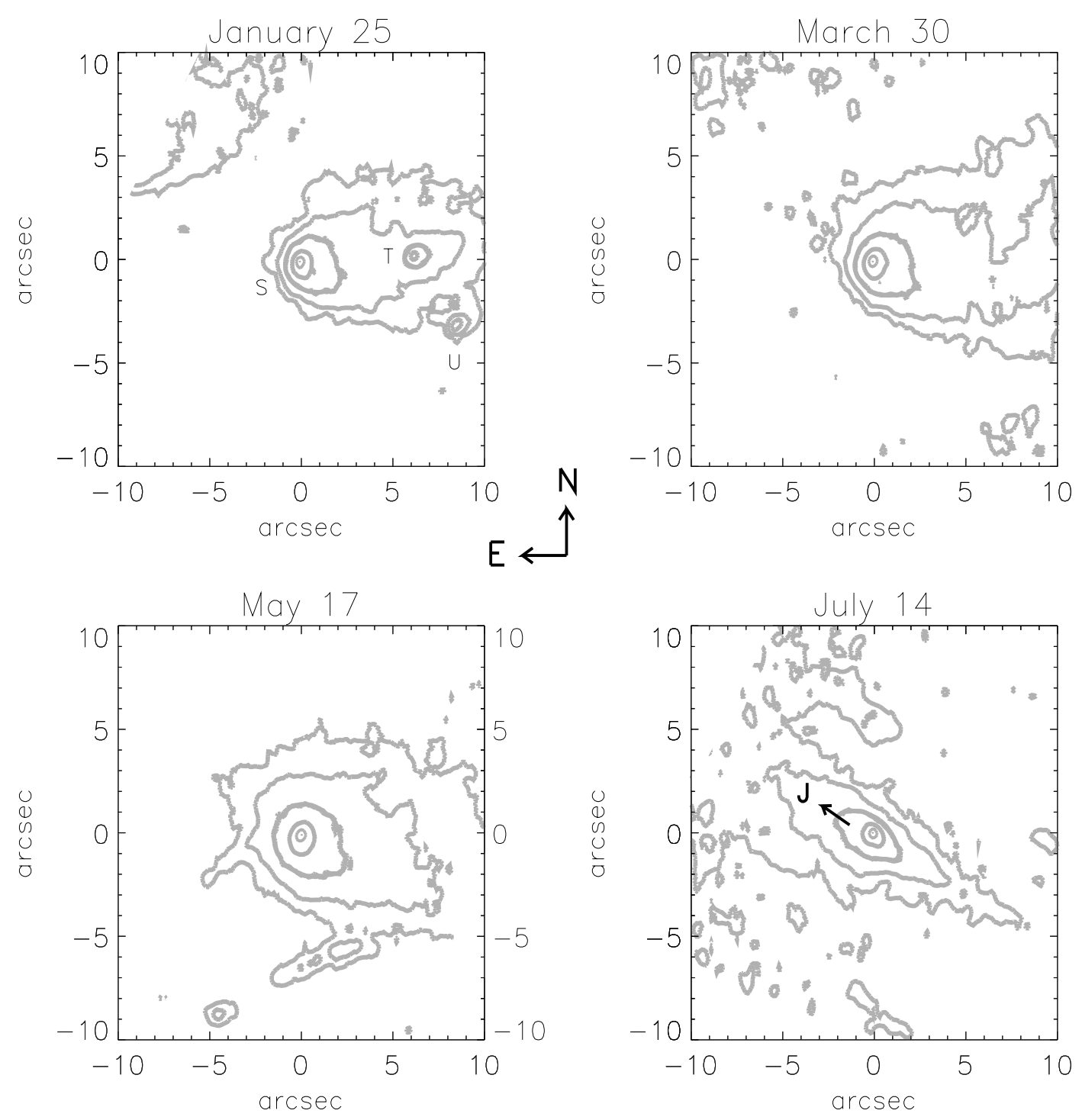

Fig. 11.- 
January 25

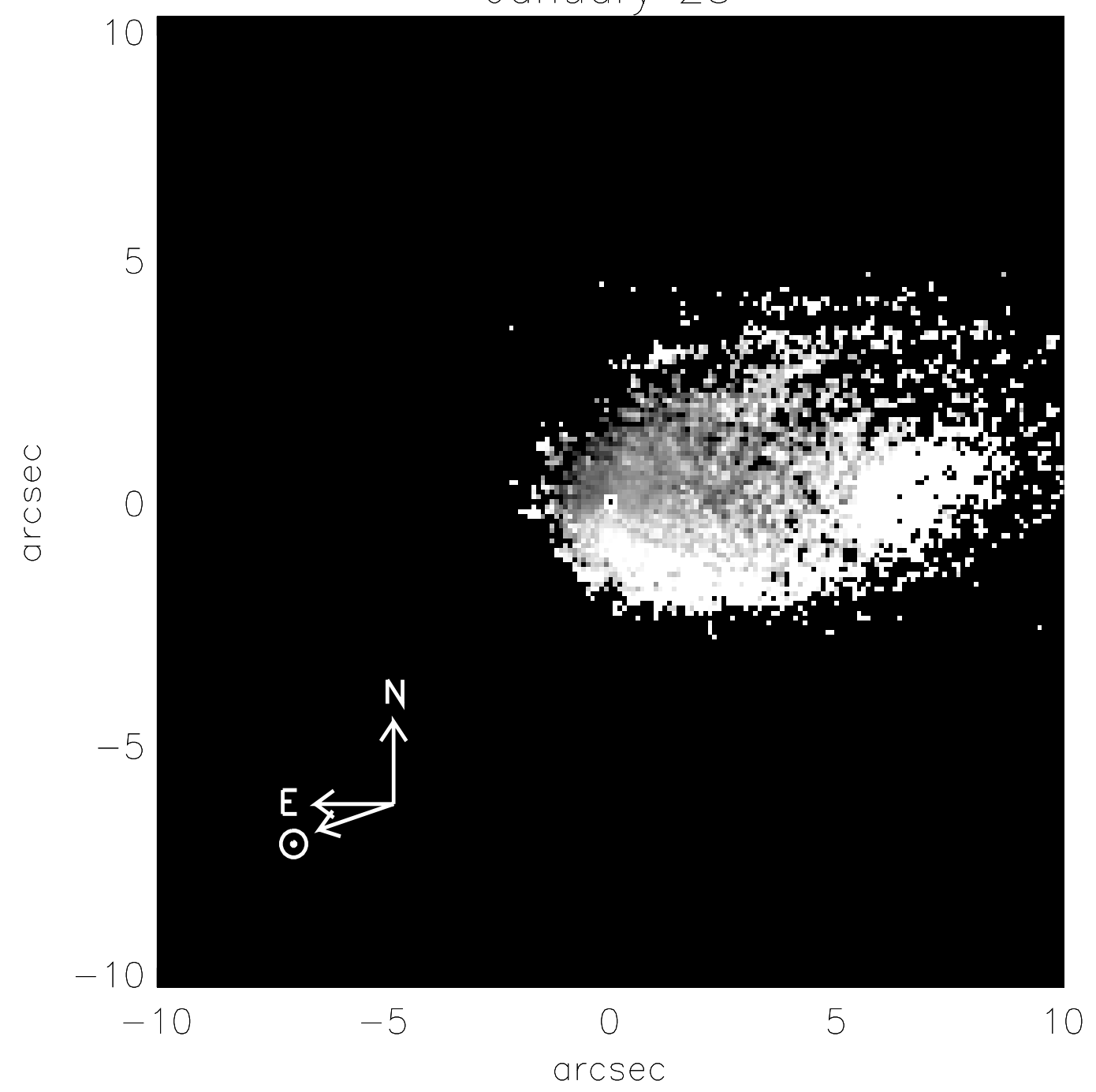

Fig. 12.- 